\title{
summary
}

\section{Patients with anterior disk displacement improve with minimal treatment}

\begin{abstract}
Minakuchi H, Kuboki T, Malsuka Y, Maekawa K, Yatani H, Yamashita A. A randomized controlled evaluation of non-surgical treatments for temporomandibular joint anterior disk displacement without reduction. J Dent Res 2001; 80:924-928
\end{abstract}

Question: Is minimal treatment effective in people who have anterior disk displacement?

Objective To evaluate non-surgical treatments of anterior disk displacement without reduction (ADDwor).

Design Randomised controlled trial (RCT) in a hospital setting. Intervention A total of 69 Magnetic Resonance Imaging (MRI) confirmed ADDwor subjects were randomly assigned to either a control group (C) that received explanation and advice, a self-care group (SC) who received a nonsteroidal anti-inflammatory drug (25 mg diclofencac tds) and self-care protocol, or a third group (PM) that received in addition the fitting of an occlusal appliance and intermittent manual jaw mobilisation therapy. Calibrated examiners collected data at the initial interview and at 0,2, 4 and 8 weeks.

Outcome measures Maximum mouth opening, visual analogue scale of pain (VAS-pain), and daily activity limitation (DAL).
Results At the 8-week point, within-group improvements were present for all variables for all groups. Between-group differences were not highly evident (see Table 1).

Conclusion The results suggest that ADDwor subjects will normally improve with only minimal treatment intervention. No significant differences were evident for the treatments tested against the control. The gradual reduction of signs and symptoms in ADDwor in treatment and control groups seems to be more related to the passage of time rather than the type of treatment.

\section{Evidence-Based Dentistry (2002) 3, 69. doi:10.1038/ sj.ebd. 6400113}

Address for reprints: H Minakuchi, Department of Fixed Prosthodontics, Okayama University Dental School, 2-5-I Shikata-cho, Okayama. Japan. E-mail: shajime@md.okayama-u.ac.jp.

Table 1 Effect of intervention* in treatment groups

\begin{tabular}{|c|c|c|c|c|c|c|}
\hline & \multicolumn{2}{|c|}{ Control } & \multicolumn{2}{|c|}{ SC } & \multicolumn{2}{|c|}{ PM } \\
\hline & Initial & 8 weeks & Initial & 8 weeks & Initial & 8 weeks \\
\hline Maximum mouth opening & 32.5 & 38.5 & 32.4 & 38.1 & 29.1 & 39.8 \\
\hline DAL score & 6.0 & 3.8 & 5.6 & 3.3 & 6.1 & 5.1 \\
\hline VAS-pain at rest & 10.8 & 7.3 & 11.4 & 6.4 & 12.4 & 3.8 \\
\hline VAS-pain during mastication & 59.0 & 29.0 & 55.8 & 24.6 & 47.7 & 26.2 \\
\hline
\end{tabular}

*Standard deviation available in original paper.

DAL, Daily activity limitation; VAS, visual analogue scale.

\section{Commentary}

This is a meticulously planned trial in a common and typical group of patients suffering temporomandibular joint pain. Subjects were only entered into the trial with MRI evidence of anterior disc displacement. They were allocated randomly to the three groups as described, one of which was a control and two that were typical conservative treatments used for this condition. Exclusion criteria were agreed before the trial started and dropouts from the trial were all accounted for. Three parameters were used to assess the progress of each patient during a 2 -month period at five timepoints. Two of the parameters were subjective, VAS and DAL, and these were measured by one trained and blinded examiner. The third parameter was objective, to measure maximum comfortable mouth opening, and active and passive ranges of motion. This involved three trained examiners using standard tests using a pressure algometer, all blinded to the treatment groups. There was a detailed description of each method used.

The results of the trial showed that all groups significantly improved in their signs and symptoms with time but, in all the parameters studied, there was no significance in the rate or extent of the improvement recorded. This included controls, where patients had been given only a detailed explanation of the condition and told of the self-limiting nature of the signs and symptoms.

This paper is an admirable example of how a RCT can and should be carried out for a common condition.

Richard Juniper

Department of Postgraduate Medical \& Dental Education, Oxford, UK 